\title{
Radio Resource Management and Interference Analysis for Downlink OFDMA in LTE
}

\author{
P.Balasundaram \\ VIT University \\ SENSE, VIT \\ University, Vellore, \\ Tamilnadu, India.
}

\author{
S.Nandakumar \\ VIT University \\ SENSE, VIT \\ University, Vellore, \\ Tamilnadu, India.
}

\author{
J.Ajanthkumar \\ VIT University \\ SENSE, VIT \\ University, Vellore, \\ Tamilnadu, India.
}

\author{
K.G.Lingesh \\ VIT University \\ SENSE, VIT \\ University, Vellore, \\ Tamilnadu, India.
}

\begin{abstract}
In $4 \mathrm{G}$ technology especially LTE relaying and orthogonal frequency division multiple access (OFDMA) are widely accepted techniques in downlink. To obtain ubiquitous coverage with high data rate to user terminals even in difficult channel conditions (e.g. cell edge) a centralized RRM algorithm has been proposed for cellular fixed relay networks in downlink OFDMA. The proposed method provides the fairness for user with minimal impact on network throughput. The average user throughput has been calculated for various bit error rate in this method. This method is queue aware and jointly performs three functions namely dynamic routing, fair scheduling and load balancing among cell nodes. This method is different from others because of its low level of complexity. Interference is analyzed between CDMA - DS base station and OFDMA base station. Additional isolation required and path losses are calculated when the two stations are not co-sited.
\end{abstract}

\section{Keywords}

OFDMA, CDMA-DS, RRM, relaying, routing, scheduling, load balancing, Additional isolation, path loss.

\section{INTRODUCTION}

Long Term Evolution is developed by (3GPP) Third Generation Partnership Project and considered to be $4 \mathrm{G}$ wireless broadband technology. LTE is defined for more frequency bands than any other technology. It uses a number of defined channel bandwidth between 1.25 and $20 \mathrm{MHz}$ LTE provides a throughput of 50 Mbps during uplink and 100 Mbps during downlink [13]. It reduces latency and increases spectral efficiency and compatible with other previous technologies. LTE will be deployed within the span of a decade its system architecture is simple which makes operating cost to be low.

Table 1. LTE specifications

\begin{tabular}{|l|l|}
\hline Downlink & OFDMA \\
\hline Uplink & SC-FDMA \\
\hline Modulation & QPSK, 16 QAM, 64 QAM \\
\hline Duplexing & FDD, TDD \\
\hline Air interface & E-UTRAN \\
\hline
\end{tabular}

OFDMA is frequency selectivity enabled multiuser diversity technique. OFDMA is a multiple access technique in OFDM and works in $2-4 \mathrm{GHz}$ band. Each user is assigned with a subset of subcarriers (sub-channels) at any time. OFDMA possess some features such as great immunity against multipath distortion, high spectral efficiency and low cost baseband processing. It is applied in Wimax and LTE.

RRM performs the functions of efficient usage of radio resource according to the available technique adapted and also provides the users QOS [8]. The use of radio resource is of greater concern in the domain of mobile communication. So the allocation of channels to the users has to be made effectively. For downlink LTE, a centralized RRM algorithm has been proposed in this paper using OFDMA and cellular fixed relay networks.

Table 2. RRM functions at layers

\begin{tabular}{|c|c|}
\hline \multirow{4}{*}{ Layer 3 } & QOS management \\
\cline { 2 - 2 } & Admission control \\
\cline { 2 - 2 } & Persistent scheduling \\
\hline
\end{tabular}

\begin{tabular}{|c|c|}
\hline \multirow{4}{*}{ Layer 2 } & $\begin{array}{c}\text { Hybrid Adaptive Repeat and } \\
\text { Request (HARQ) }\end{array}$ \\
\cline { 2 - 2 } & Dynamic scheduling \\
\cline { 2 - 2 } & Link adaptation \\
\hline
\end{tabular}

\begin{tabular}{|c|c|}
\hline \multirow{4}{*}{ Layer 1 } & $\begin{array}{c}\text { Physical Downlink Control Channel } \\
\text { adaptation(PDCCH) }\end{array}$ \\
\cline { 2 - 2 } & Channel Quality Indicator(CQI) \\
\cline { 2 - 2 } & Power control \\
\hline
\end{tabular}

RRM algorithm is based on queue aware and the functions such as scheduling, dynamic routing and load balancing are jointly performed by it. The information about wireless link capability or the remaining capacities are collected by RRM function. The basic RRM functions are performed at various layers. Layer 3 is semi dynamic mainly executed during setup of new data flows. Layer 2 is dynamic new actions conducted every Transmission Time Interval (TTI). Layer 1 is fast dynamic. 
The high data rate is obtained even at the cell edge by the users due to the combined operation of relay and OFDMA. This leads to ubiquitous coverage in the cell. High degree of user fairness and intra-cell, load balancing [1] has been obtained. The opportunities provided in channel dynamism, spatial diversities have been utilized.

Many queue/traffic aware fair scheduling algorithms have been proposed in conventional cellular networks and few among them are

a. Channel State Dependent Packet Scheduling

b. Channel Independent packet Fair-Queuing (CIF-Q) [11].

c. OFMDA based algorithms [2]-[3].

The algorithms cannot be applied directly to relay-enhanced networks because it provides solution only to scheduling. The fairness awareness schemes such as Proportional Fair Scheduling (PFS) [4] which depend on achievable and allocated capacities, does not provide the desired fairness.

\section{DESCRIPTIONS AND ASSUMPTIONS IN SYSTEM}

Let ' $U$ ' be the number of user terminals(UT), B be the base station(BS), E be the number of relay stations(RS) and ' $S$ ' be the number of sub-channels. The total bandwidth is divided into ' $\mathrm{S}$ ' number of sub-channels and each sub-channel is further divided into ' $\mathrm{L}$ ' subcarriers.

Assign ' $U$ ' user buffers at B and each of E Relay stations in a cell. Based on the traffic model the user packets arrive at corresponding ' $\mathrm{B}$ ' buffer. In the particular frame duration channel fading is considered to be time invariant. Two modes of operation have been considered (in this paper).

1) Open routing

2) Constrained routing

Open routing deployment any user can be connected to any combination of ' $E$ ' Relay stations only in two hops.

Constrained mode of routing a user terminal can receive from a group of nodes (B or E) \& any node can transmit to many destinations simultaneously using different orthogonal subchannels. This mode provides substantial savings in feedback overhead.

Using orthogonal sub-channels any Relay station can concurrently receive and transmit. Two antennas have been deployed for the fixed Relay stations.

$\begin{array}{ll}\text { Directional antenna } & : \text { BS } \stackrel{\text { Feeder link }}{\longrightarrow} \text { RS } \\ \text { Omni directional } & : \quad \text { RS } \longrightarrow \text { All UT's antenna }\end{array}$

Load balancing is performed by providing equal distribution of subcarriers among the nodes [5]. Balancing the traffic load reduces the packet processing delays at the regenerative relays.

\section{RADIO RESOURCE ALLOCATION AT BASE STATION}

By maintaining throughput fairness among users, the process of maximizing the total cell throughput is carried out. Throughputoptimal scheduling policy which stabilizes user queues at all nodes in a system that receives equal inelastic arrival rates at BS using two hops at most is operated [11].
The RRA scheme needs to assign the sub-channels with the highest capacities at any node to achieve the maximum total cell throughput. Radio resource at BS can be allocated using

a. Binary Integer Linear Programming (BILP)

b. Hungarian algorithm (proposed)

Table 3. Complexity comparison of RRA algorithms

\begin{tabular}{|c|c|}
\hline Allocating scheme & Complexity \\
\hline BILP & $\mathrm{O}\left(((\mathrm{E}+1) \mathrm{U})^{\mathrm{S}}\right)$ \\
\hline Hungarian algorithm & $\mathrm{O}\left(\mathrm{S}^{3}\right)$ \\
\hline
\end{tabular}

\section{PROPOSED \\ ALGORITHM}

HUNGARIAN

The proposed algorithm [6] is an efficient solver and even used in various scheduling algorithms for non-relaying networks. Let us define demand metric ' $F$ ' for any node $e_{e}-U_{u}$ link on subchannel ' $S$ ' as the product of the achievable rate on that access link and queue length of user's buffer at that node and the demand of the feeder link on sub-channel ' $S$ ' incorporates the queues at $\mathrm{BS}$ (node 0 ) and those at $\mathrm{RS}_{\mathrm{e}}$ and given by

$$
\begin{array}{ll}
\mathrm{F}_{\mathrm{s}, \mathrm{e} \rightarrow \mathrm{u}}=\mathrm{Y}_{\mathrm{e}, \mathrm{u}, \mathrm{s}} \mathrm{Z}_{\mathrm{u}}^{\mathrm{e}} & \mathrm{e}=0,1 \ldots, \mathrm{E} \\
\mathrm{F}_{\mathrm{s}, 0 \rightarrow \mathrm{e}}=\mathrm{Y}_{0, \mathrm{e}, \mathrm{s}} \max _{\mathrm{u}}\left\{\left(\mathrm{Z}_{\mathrm{u}}^{0}-\mathrm{Z}_{\mathrm{u}}^{\mathrm{e}}\right)^{+}\right\} & \mathrm{e} \neq 0
\end{array}
$$

function (.)+ sets negative argument to zero.

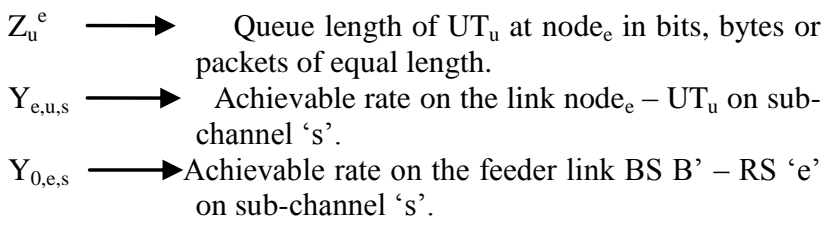

These rates are calculated by using continuous rate formula for adaptive modulation and coding (AMC) under the condition of no loss of generality.

$\mathrm{Y}_{\mathrm{i}, \mathrm{j}, \mathrm{s}}=\mathrm{C} \log 2\left(1+\left(\frac{-1.5 \mathrm{Gi}, \mathrm{j}, \mathrm{s}}{\ln (5 \mathrm{P})}\right)\right)$

$\mathrm{G}_{\mathrm{i}, \mathrm{j}, \mathrm{s}} \longrightarrow$ Received signal to interference plus noise ratio (SINR) from source $\mathrm{i}$ at destination $\mathrm{j}$ on subchannel ' $\mathrm{S}$ ' considering all the dominant interference observed in the previous transmission.

$\mathrm{P} \longrightarrow$ Target Bit error rate

$\mathrm{C} \longrightarrow$ OFDM sub-channel bandwidth

1. Calculate the $(E+1)$ demand metrics on each sub-channel and apply Hungarian algorithm to $\mathrm{Sx}(\mathrm{E}+1)$ demand matrix $\left|F_{s, e}\right|$ (shown in Table IV.1).

2. On each sub-channel find the lowest demand metric and subtract it from the remaining values (Shown in Table IV.2).

3. If there results any zero in each row, then mark the zero and assign the particular sub-channel to that corresponding Relay stations / Base station.

4. Check whether all Relay station / Base station have an assigned sub-channel. 
5. If a particular Relay station / Base station doesn't have assigned then consider that corresponding column and find the minimum value and subtract it from other values in that column.

6. Even then, still there is any Relay station / Base station doesn't have an assigned sub-channel.

7. Then follow the given steps initially, assign as many RS / BS as possible

a. Mark all sub-channels that are unassigned

b. Then mark all zeros in that sub-channel

c. Then find any assignment to that particular RS / BS and encircle over the whole particular RS / BS.

d. Mark that corresponding sub-channel to that particular RS / BS

e. Select all sub-channels that were unmarked(Shown in Table IV.3)

f. From the remaining elements that were not encircled find the minimum value and subtract it from the elements that were considered.

g. This results a value zero which indicates a new assignment of sub-channel to a particular RS / BS

h. Repeat this procedure till all RS / BS are assigned with a sub-channel

i. All sub-channels were assigned (shown in Table IV.4)

This proposed algorithm solves a one-one optimization problem by doing S / (E+1) times (iterations). The affected UT's queue gets virtually updated based on the decisions of the previous iteration.

$\mathrm{Z}_{\mathrm{u}}^{\mathrm{e}(\mathrm{i}+1)}=\left(\mathrm{Z}_{\mathrm{u}}^{\mathrm{e}(\mathrm{i})}-\left|\mathrm{Y}_{\mathrm{e}}^{\mathrm{i}} \mathrm{T}\right|\right)^{+}$

Dynamic Routing uses the maximum differential backlog indicated by $\max _{\mathrm{u}}\left\{\mathrm{Z}_{\mathrm{u}}{ }^{0}-\mathrm{Z}_{\mathrm{u}}{ }^{\mathrm{e}}\right\}$ to establish the router. Routing is performed dynamically $\&$ jointly with its resource allocation.

\section{INTERFERENCE BETWEEN BASE STATIONS}

Consider CDMA-DS base station as interference source and OFDMA as victim. Different cases of CDMA-DS base stations are put forward for the analysis. The interference sources are considered to be operating in macro cellular, micro cellular and pico cellular networks [9] while receiver base station is considered to be operating in macro cellular networks. The level of interference received depends on spectral leakage of the interferer's transmitter and adjacent channel performance of the receiver while the former is characterized by Adjacent Channel Leakage (ACLR) and latter by Adjacent Channel Selectivity (ACS).

Additional isolation $=\mathrm{L}-\mathrm{M}$

$\mathrm{L} \longrightarrow$ Level of interference received

$\mathrm{M} \longrightarrow$ Maximum interference limit

Adjacent Channel Interference Ratio (ACIR) gives the combined value of ACLR and ACS. Maximum interference limit values of OFDMA and CDMA-DS are $-110 \mathrm{dBm}$ and -109 $\mathrm{dBm}$ respectively.

\section{PATH LOSS}

Let OFDMA base station and CDMA-DS base stations are not co-sited and placed at various distances.

Case 1:

Path loss is calculated between macro cellular CDMA-DS and OFDMA base stations for various distances by considering the propagation model to be free space [10].

$\mathrm{PL}(\mathrm{dB})=40.7+20 \log _{10}(\mathrm{~b})$

$\mathrm{b} \longrightarrow$ distance between the base stations.

Case 2:

Let the CDMA-DS and OFDMA base stations be in micro cellular and macro cellular networks respectively and path loss is given by

$\mathrm{PL}(\mathrm{dB})=147.2+38 \log _{10}(\mathrm{~b})$

Case 3:

Let CDMA-DS and OFDMA base stations be in pico cellular and macro cellular networks respectively. Path loss is calculated by

PL $(d B)=159.1+38 \log _{10}(b)$

\section{MODELS AND PARAMETERS - SIMULATION}

Simulated network and parameters for channels are indicated in Table 4. UTs are considered to be uniformly distributed within the cell. For BS-RS (LOS) links Rician type of fading is considered and for all other (NLOS) links Rayleigh type of fading is considered. Queues at BS are assumed to be arriving independently in the form of Poisson process.

Table 5. Parameters -simulation

\begin{tabular}{|c|c|}
\hline Parameter & Value \\
\hline BS- BS distance & $1 \mathrm{Km}$ \\
\hline RS distance from BS & 0.65 x cell radius \\
\hline UT min close in distance to BS & $35 \mathrm{~m}$ \\
\hline Carrier frequency & $2.5 \mathrm{GHz}$ \\
\hline Total bandwidth & $20 \mathrm{MHz}$ \\
\hline TDD frame length & $2 \mathrm{~ms}$ \\
\hline OFDM subcarrier bandwidth & $10.9375 \mathrm{KHz}$ \\
\hline OFDM symbol duration & $102.86 \mu \mathrm{sec}$ \\
\hline Sub-channel width & $18 \mathrm{subcarriers}$ \\
\hline Total number of sub-channels & 102 \\
\hline
\end{tabular}




\section{RESULTS AND DISCUSSION}

The average cell throughput for varying users per cell at different values of BER (Bit Error Rate) are calculated by using

$\mathrm{T}_{\mathrm{k}}=\sum_{u=1}^{U} \rho \mathrm{u}, \mathrm{s} \mathrm{Yu}, \mathrm{s}$

$\rho_{\mathrm{u}, \mathrm{s}} \longrightarrow \mathrm{u}^{\text {th }}$ UT binary assignment variable to $\mathrm{e}^{\text {th }}$ node

$\rho_{\mathrm{u}, \mathrm{s}} \quad \in \quad\{0,1\} \quad \forall(\mathrm{e}, \mathrm{u}, \mathrm{s})$

IEEE $802.16 \mathrm{~m}$ fairness index [7] is indicated by

Fairness Index $=\frac{\text { User's throughput rate }}{\text { Average throughput rate }}$

$\operatorname{FIu}(\mathrm{t})=\frac{\mathrm{Tu}(\mathrm{t})}{\frac{1}{\mathrm{U}} \sum_{\mathrm{i}=1}^{\mathrm{U}} \operatorname{Ti}(\mathrm{t})}$

$\mathrm{FIu}(\mathrm{t}) \longrightarrow$ Fairness Index

$\mathrm{Tu}(\mathrm{t}) \longrightarrow$ User's throughput rate

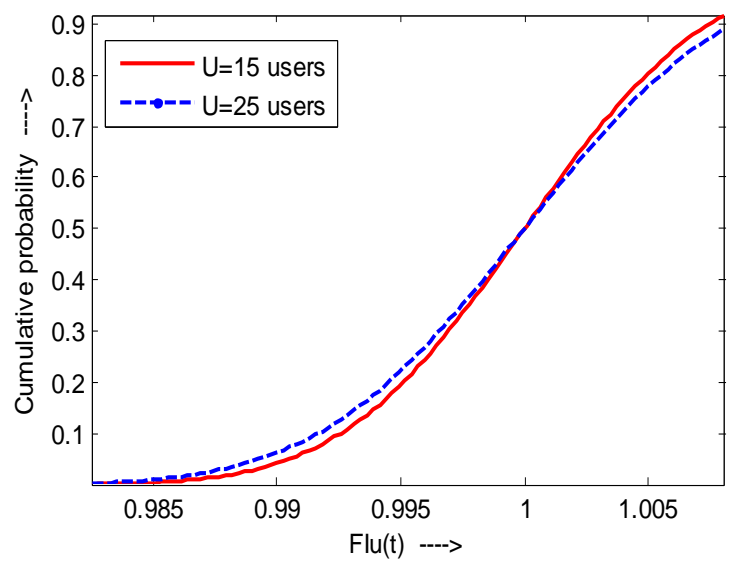

Fig 1.Average Cell Throughput

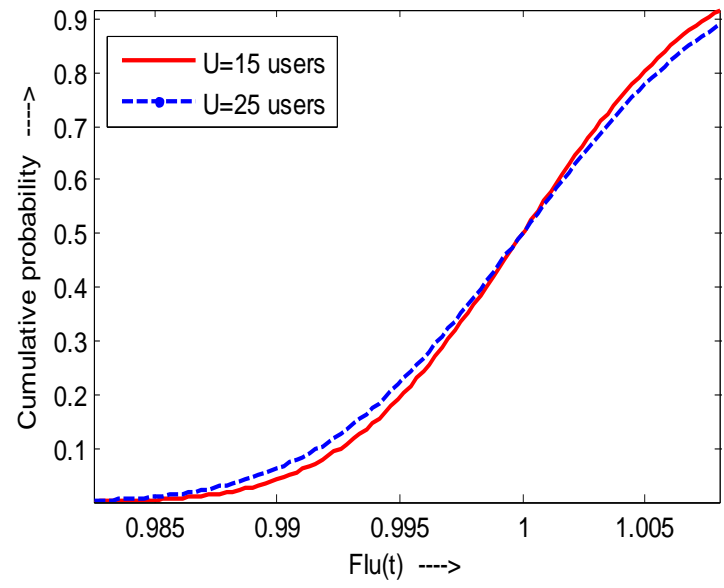

Fig 2. Fairness Index CDF for 15 and 25 UT'S / Cell
In Fig.1, for various bit error values, the average cell throughput value increases as the number of users per cell increases. It maximizes total cell throughput by exploiting the diversities such as frequency, traffic, multi user and spatial. The fairness index is used to determine whether users are receiving fair share of resources [12]. For 15 users and 25 users the time average throughput rates have been calculated and used to plot CDF as shown in Fig. 2. As the number of users per cell gets increased its difficult to maintain fairness.

In Fig.3, when both of the base stations are macro cellular, additional isolation power required is higher but as distance between them increases the required value gets decreased for coexistence. The absolute value of additional isolation power value signifies the amount of margin available in the adjacent channel protection. When both source and receiver base stations are in micro cellular and macro cellular networks the additional isolation power required decreases to negative value as distance increases. When the source and receiver base stations are in pico cellular and macro cellular networks the additional power required are getting increased in the negative value as distance increases. The negative value of additional isolation power indicates that the isolation provided by the equipment is sufficient to limit the interference to acceptable level.

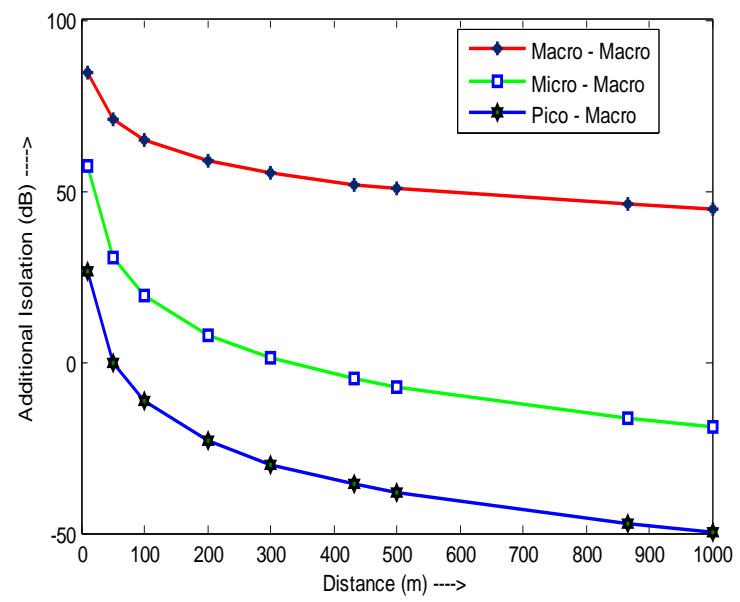

Fig.3.Additional Isolation for OFDMA

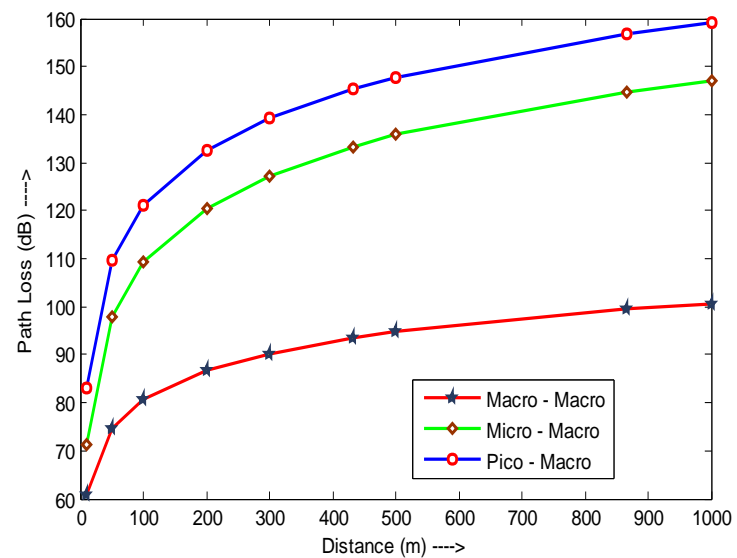

Fig 4.Path Loss between interference source and victim 
In Fig.4, for different cases the path loss gets increased as distance between the base stations gets increased. The path losses when both base stations are macro cellular are lesser when compared to the case of base stations to be in micro cellular - macro cellular and pico cellular - macro cellular.

\section{CONCLUSION}

The proposed scheme provides fairness aware joint routing and scheduling in cellular environment. It provides ubiquitous coverage, cell edge throughput, fairness and load balancing which makes it superior over other previous methods irrespective of geographical deployment by exploiting the opportunities in frequency, spatial and traffic diversities. Additional isolation is calculated to provide an indication of the size of the guard band that would be required.

\section{REFERENCES}

[1] Mohamed Salem, Mahmudur Rahman, "Fairness-Aware Radio Resource Management in downlink OFDMA Cellular Relay Networks", IEEE transactions on wireless Communications, Vol.9, no. 5. May 2010.

[2] P. Parag , S. Bhashyam and R. Arvind , "A subcarrier allocation algorithm for OFDMA using buffer and channel state information," in Proc. IEEE veh. Technol. Conf., pp. 622-625, Sep. 2005.

[3] M. Kobayashi and G. Caire, "Joint beamforming and scheduling for a multi-antenna downlink with imperfect transmitter channel knowledge," IEEE J. Sel. Areas Commun., vol.25, no.7, pp.1468-1477, Sep-2007.

[4] H. Kim and Y. Han, "A proportional fair scheduling for multicarrier transmission systems," IEEE Commun. Lett., vol.9, no.3, pp. 210-212, Mar.2005
[5] C. Bae and D.H. Cho, "Fairness-aware adaptive resource allocation scheme in multihop OFDMA systems," IEEE Commun., Lett., vol. 11, no.2 , pp. 134-136, Feb 2007.

[6] H.W.Kuhn, "The Hungarian problem for the assignment problem", Naval Research Logistic Quarterly, vol.2, no.1pp.83-97, 1955

[7] IEEE C802.16m-07/306, "Protocol structure to support cooperative transmission", IEEE 802.16 Broadband Wireless Access Working Group.

[8] Evolved Universal Terrestrial Radio Access(E-UTRA) requirements for radio resource management (3GPP TS 36.133 version 9.4.0 Release 9)

[9] ITU-R M.2146 "Coexistence between IMT-2000 CDMADS and IMT 2000 OFDMA" (5/2009).

[10] ITU-R M.2039 "Characteristics of terrestrial IMT-2000 systems for frequency sharing / interference analyses" $(11 / 2010)$.

[11] Z.Han and K.J.Liu, "Resource Allocation for Wireless Networks: Basics, Techniques, and Applications", Cambridge University (2008).

[12] L. Xiao and L. Cuthbert, "Improving fairness in relaybased access networks,"in Proc. 11th International Symp. Modeling, Analysis Simulation Wireless Mobile Syst., $(10 / 2008)$.

[13] Harri Holma and Antti Toskala, "LTE for UMTS OFDMA and SCFDMA radio access," 2009, John Wiley and Sons Ltd. 